\title{
La performance periodística en la cobertura deportiva: un estudio comparado de televisión, radio, prensa impresa y medios online en Chile
}

\section{Andrés Scherman ${ }^{1}$ Claudia Mellado²}

Recibido: 14/03/2018

Aprobado por pares: 19/06/2018
Enviado a pares: 09/04/2018

Aceptado: 21/09/2018

DOI: $10.5294 /$ pacla.2019.22.3.8

Para citar este artículo / to reference this article / para citar este artigo

Scherman, A. y Mellado, C. (2019). La performance periodística en la cobertura deportiva: un estudio comparado de televisión, radio, prensa impresa y medios online en Chile.

Palabra Clave, 22(3), e2238. DOI: http://doi.org/10.5294/pacla.2019.22.3.8

\section{Resumen}

Tradicionalmente se ha cuestionado el periodismo deportivo, acusándolo de falta de rigor, escasa capacidad crítica con las fuentes y de ignorar los conflictos sociales. Este artículo busca establecer si existen diferencias en las características de la cobertura noticiosa que realizan las secciones de deportes y la que efectúan otras secciones de los medios, evaluando aspectos como cantidad de fuentes, diversidad de puntos de vista y entrega de información verificable. Además, se indaga si existen diferencias en la presencia de los roles profesionales (intervencionista, vigilante, leal, servicio, infoentretenimiento y cívico) en las noticias de las secciones de deportes y las generadas por otras áreas. Utilizando como metodología de investigación un análisis de contenido de noticias realizado en Chile $(\mathrm{n}=2,118)$, durante 2015 en prensa escrita, online, televisión y radio, este estudio comparó las características de la cobertura dada a las noticias de la sección de deportes

1 orcid.org/0000-0002-7982-1738. Universidad Diego Portales, Chile. andresscherman@udp.cl

2 orcid.org/0000-0002-9281-1526. Pontificia Universidad Católica de Valparaíso, Chile. claudia.mellado@pucv.cl 
con las notas publicadas en otros frentes informativos. Los resultados tienden a confirmar las miradas más críticas sobre el periodismo deportivo y muestran que, en comparación con las noticias de política, el número de fuentes que se consulta, la diversidad de puntos de vista que se muestran y la cantidad de información verificable que recibe la audiencia son menores. Respecto de la presencia de los roles profesionales, se observa que, en comparación con otras secciones, las noticias deportivas incluyen con mayor frecuencia roles relacionados con la entrega de opiniones de los periodistas que expresan una mirada positiva de las fuentes.

\section{Palabras clave (Fuente: tesauro de la Unesco)}

Periodismo; prácticas periodísticas; roles profesionales; deportes; Chile; multiplataforma; radiodifusión; televisión; prensa; radio. 


\section{Journalistic Performance in Sports Coverage: A Comparative Study of Television, Radio, Press, and Online Media in Chile}

\section{Abstract}

Traditionally, sports journalism has been questioned for lacking rigor, having poor critical thinking skills regarding sources, and ignoring social conflicts. This article seeks to establish whether there are differences in the characteristics of news coverage between sports and other sections of media, assessing aspects such as number of sources, diversity of viewpoints, and delivery of verifiable information. In addition, it is inquired if there are differences in the presence of professional roles (interventionist, watchdog, loyal facilitator, service journalism, infotainment and civic-oriented roles) between sports news and those from other sections. Using as a research method an analysis of news contents in Chilean press, online media, television and radio $(\mathrm{n}=2.118)$ in 2015 , this study compared the characteristics of news coverage in the sports section with news published in other areas. Results tend to confirm the most critical views on sports journalism and show that, compared to political news, the number of sources used, the diversity of viewpoints involved, and the amount of verifiable information received by the audience are small. As to the presence of professional roles, it is observed that, compared to other sections, sports news more frequently includes roles related to journalists' opinions expressing a positive view of sources.

\section{Keywords (Source: Unesco Thesaurus)}

Journalism; journalistic practices; professional roles; sports; Chile; multiplatform; broadcasting; television; press; radio. 


\section{A performance jornalística na cobertura esportiva: um estudo comparado de televisão, rádio, imprensa impressa e meios on-line no Chile}

\section{Resumo}

De forma tradicional, questiona-se o jornalismo esportivo acusando-o de falta de rigor, pouca capacidade crítica com as fontes e de ignorar os conflitos sociais. Este artigo pretende estabelecer se existem diferenças nas características da cobertura jornalística que as seções de esporte realizam e a que outras seções dos meios efetuam, avaliando aspectos como quantidade de fontes, diversidade de pontos de vista e entrega de informação verificável. Além disso, questiona-se se existem diferenças na presença dos papéis profissionais (intervencionista, vigiante, leal, serviço, infotenimento e cívico) nas notícias das seções de esportes e nas geradas por outras áreas. Utilizando como metodologia de pesquisa uma análise de conteúdo de notícias realizada no Chile ( $\mathrm{n}=2.118)$, durante 2015 , em imprensa escrita, on-line, televisão e rádio, este estudo comparou as características da cobertura dada às notícias da seção de esportes com as publicadas em outras fontes informativas. Os resultados tendem a confirmar as visões mais críticas sobre o jornalismo esportivo e mostram que, em comparação com as notícias de polícia, o número de fontes consultadas, a diversidade de pontos de vista e a quantidade de informação verificável que a audiência recebe são menores. A respeito da presença dos papéis profissionais, observa-se que, em relação com outras seções, as notícias esportivas incluem, com maior frequência, papéis relacionados com opiniões dos jornalistas que expressam um olhar positivo das fontes.

\section{Palavras-chave (Fonte: tesauro da Unesco)}

Jornalismo; práticas jornalísticas; papéis profissionais; esportes Chile; multiplataforma; radiodifusão; televisão; imprensa; rádio. 


\section{Introducción}

Hasta ahora, el estudio de los roles profesionales que cumplen los periodistas se ha concentrado en especial en el ámbito político, dejando atrás otras áreas informativas (Mellado et al., 2017; Zelizer, 2017), que, si bien no son consideradas parte del periodismo mainstream o de batalla, están entre las más leídas o vistas, como la sección de deportes (Billings, 2010; Cushion 2012; Díaz y Mellado, 2017; Valenzuela y Arriagada, 2009). El predominio de una mirada normativa respecto del periodismo por parte de los investigadores a nivel mundial, quienes se han concentrado en estudiar los roles que el periodismo debe cumplir más que en el análisis de cómo funcionan efectivamente los medios de comunicación, ha terminado opacando el estudio y la comprensión de ámbitos noticiosos alejados de la política o el control del poder de las autoridades. De esta manera, se ha generado una suerte de círculo vicioso en que áreas temáticas como deportes o entretención son consideradas "mal periodismo", lo que finalmente hace imposible comprender cómo funcionan estos espacios informativos - muy altos en popularidad - y qué tipo de roles periodísticos predominan en la información que entregan.

Las secciones de deportes generan una gran cantidad de noticias en los medios de comunicación, teniendo una importancia comercial relevante en la estructura de financiamiento de los medios (Boyle, 2014). En la investigación sobre esta área de trabajo periodístico, ha predominado una mirada crítica, que se puede sintetizar en el concepto de departamento de juguetes de los medios noticiosos (toy department of news media). De acuerdo con este planteamiento, la sección de deportes de los medios es un lugar en que predomina la frivolidad, las prácticas profesionales están alejadas del rigor y los periodistas no toman distancia de las fuentes informativas, perdiendo capacidad crítica sobre ellas.

Este estudio busca analizar si las noticias generadas en las secciones deportivas de los medios de comunicación, en distintas plataformas mediáticas, presentan características significativamente distintas de las que posee la cobertura noticiosa de otras secciones. Específicamente, se persiguen dos objetivos: a) establecer si las noticias deportivas presentan estilos de repor- 
teo distintos de los de otras secciones de los medios (número de fuentes, diversidad de puntos de vista y presencia de información verificable), yb) establecer si existen diferencias significativas entre los roles periodísticos que se ponen en práctica en la cobertura de la sección de deportes y los roles que se materializan en otras secciones de los medios.

El artículo está organizado de la siguiente forma. En la primera parte, se revisa la discusión sobre las características del periodismo deportivo y se explican los conceptos de rol profesional y de performance periodística, que posteriormente serán medidos y analizados en el desarrollo de la investigación. En segundo lugar, y utilizando un análisis de contenido realizado en Chile durante 2015, se revisan las características del trabajo periodístico efectuado en las secciones de deportes de los distintos medios que componen la muestra de estudio, y se lo comparará con lo que sucede en otras secciones noticiosas. Por último, se discuten las contribuciones que implican los hallazgos de este estudio para la investigación sobre el periodismo deportivo y para los estudios sobre roles profesionales y role performance.

\section{Periodismo deportivo: ¿toy department?}

Tradicionalmente, el periodismo deportivo ha sido criticado por no cumplir los estándares tradicionales de la profesión como objetividad, independencia o ejercer el control de los grupos de poder (Márquez-Ramírez \& Rojas, 2017; Salwen \& Garrison, 1987; Surface, 1972). Por estos motivos, en general se plantea que el periodismo deportivo no cumple con la función de ser un "cuarto poder" o "perro guardián", que realiza un escaso aporte a la construcción de la democracia en la esfera pública y que se concentra, principalmente, en la cobertura de eventos que están previstos y que no constituyen sorpresa alguna (Pew Research Center: Journalism \& Media staff, 2005; Márquez-Ramírez \& Rojas, 2017). Incluso algunos autores sostienen que, en los medios de comunicación, la sección de deportes constituye el "el departamento de juguetes" (the toy department of news media), ya que en ella se trabajaría con cierta frivolidad y con menor independencia que en otras secciones de los medios (English, 2017; Garrison \& Salwen, 1989; Rowe, 2007). Producto de esta visión crítica, el periodismo 
deportivo es escasamente nombrado cuando se discute sobre periodismo de excelencia (Weedon, Wilson, Yoon \& Lawson, 2018).

Las críticas al periodismo deportivo también se extienden a su poca rigurosidad, lo que se traduciría en un uso frecuente de rumores y una separación poco clara entre información y las opiniones entregadas por los periodistas. En esta misma línea, se cuestiona el bajo número de fuentes que se utiliza, en comparación con lo realizado por otras secciones temáticas de los medios (Gómez, 2013; Márquez-Ramírez \& Rojas, 2017).

Uno de los principales cuestionamientos que ha recibido el periodismo deportivo es su falta de independencia y distancia de las fuentes informativas. Se discute que los profesionales del sector terminan, muchas veces, desempeñando el papel de hinchas más que el de periodistas, transformándose en verdaderos promotores de los equipos, los deportistas y las industrias deportivas que deben cubrir (Boyle, 2017; English, 2017; Márquez-Ramírez \& Rojas, 2017; Rowe, 2007). Incluso, se ha llegado a plantear que el periodismo deportivo opera como una empresa publicitaria, debido a la positiva cobertura que realiza de los eventos deportivos y a la omisión de aspectos controvertidos vinculados con el dinero, la política o el impacto social del deporte (Rowe, 2007, Schultz-Jorgensen, 2005). Esta falta de independencia con las fuentes se ha ejemplificado, por ejemplo, con lo que sucedió en Australia durante 2015 cuando los periodistas que cubrían los Juegos de la Commonwealth se tomaron selfies con el atleta jamaiquino Usain Bolt tras su conferencia de prensa (English, 2017).

Además de los aspectos mencionados, el trabajo del periodismo deportivo también ha recibido críticas por el espacio asignado a grupos de la población como las mujeres (Franks \& O'Neill, 2016; Hardin \& Whiteside, 2009) y las personas con capacidades diferentes (Solves, Sánchez \& Ruiz, 2016).

Pese a estas críticas sobre la manera en que los periodistas desarrollan su trabajo, las secciones deportivas tienen una gran relevancia en los medios de comunicación, producto de su capacidad de llegar a públicos masivos y 
de generar recursos económicos en una industria que ha visto amenazada su existencia en las últimas décadas y que ha tenido que replantear sus modelos de negocios (Márquez-Ramírez \& Rojas, 2017; Rowe, 2004, 2007; Sugden \& Tomlinson, 2007). Así es como se llega a la paradoja de que las secciones de deportes dispongan de importantes recursos para hacer su trabajo (sueldos, equipos periodísticos, viajes, etc.), pero que paralelamente sus periodistas se sientan poco apreciados y con un estatus inferior al de sus pares de otras secciones (Rowe, 2004). La importancia económica que han adquirido las noticias de deportes en los medios ha llevado a Farrington, Kilvington, Saeed y Price (2012) a plantear que esta sección pasó de ser "el departamento de juguetes" al "departamento de finanzas" de los medios, mientras que, basándose en las ideas de Pierre Bourdieu, English (2016) asegura que, dentro del campo de los medios de comunicación, el subcampo de las secciones deportivas está caracterizado por el acceso al poder económico.

La investigación empírica sobre las características del periodismo deportivo ha tenido resultados contradictorios. Mientras algunos estudios han apoyado la mirada crítica que han planteado autores como Salwen \& Garrison (1988) y Surface (1972), y otras indagaciones han mostrado resultados en las que no se aprecian diferencias relevantes entre la forma de trabajo y la cultura profesional de los periodistas deportivos y los que se desempeñan en otras secciones periodísticas.

Entre las miradas críticas está la investigación de Rowe (2007), quien tras analizar datos sobre el periodismo deportivo en Australia, recogidos por la International Sport Press Survey 2005, enumera algunas características de las noticias que aparecen en las secciones de deportes: escasa cobertura de temas relacionados con debates públicos (como asuntos de género o integración social), consulta de un reducido espectro de fuentes, baja frecuencia de mujeres que se desempeñan como periodistas deportivas y escasa cobertura de mujeres deportistas. Por estos motivos, Rowe concluye que, en este caso, se justifica la peyorativa calificación de "departamento de juguetes".

Un resultado similar al de Rowe (2007) obtuvieron Márquez-Ramírez \& Rojas (2017) tras estudiar cómo dos diarios de referencia y dos dia- 
rios especializados en deportes de España y México cubrieron el llamado FIFAGate, que es considerado el mayor escándalo del fútbol mundial. En su investigación, Márquez-Ramírez \& Rojas encontraron que los diarios especializados, en comparación con la prensa generalista, dieron menos cobertura a temas de corrupción, consultaron un menor número de fuentes por nota informativa y entregaron menos evidencia de los hechos (Márquez-Ramírez \& Rojas, 2017).

Sin embargo, otros estudios no concuerdan con que el periodismo deportivo carezca de capacidad crítica o que opere en su interior una cultura profesional distinta de la de sus pares que se dedican a cubrir otros temas. Tras estudiar 1.265 artículos sobre la cobertura de la serie de cricket entre Australia y la India en 2014 y 2015, English (2017) encontró que la mayor parte de los artículos publicados tenían una proporción más alta de elementos críticos que condescendientes. Por otra parte, durante las últimas dos décadas, los periodistas deportivos crecientemente han trabajado en la cobertura de temas más controvertidos como el uso de drogas por parte de los periodistas, la relación entre política y deportes, la violencia en el deporte, la homosexualidad de algunos deportistas y el rol de los agentes o representantes (Cassidy, 2017; Garrison \& Salwen, 1989).

Finalmente, Garrison \& Salwen (1989) encontraron que los periodistas deportivos en los Estados Unidos utilizan criterios profesionales y éticos similares al del resto de los periodistas que trabajan en los medios. A pesar de estos importantes estudios, todavía existe un vacío importante en la literatura, en especial en el ámbito de los estudios sobre prácticas profesionales específicas, como sus niveles de objetividad.

Según esta revisión de la literatura, se plantean las primeras dos preguntas de investigación de este estudio:

P1: ¿Existen diferencias significativas en las características de la cobertura noticiosa que realizan las secciones de deportes y la que efectúan otras secciones, considerando aspectos como cantidad de fuentes, diversidad de puntos de vista y entrega de información verificable? 
P2: ¿Las características de la cobertura que realizan las secciones de deportes, -considerando aspectos como cantidad de fuentes, diversidad de puntos de vista y entrega de información verificable, es similar en las distintas plataformas mediales (prensa escrita, medios online, televisión y radio) o existen diferencias significativas entre ellas?

\section{Roles periodísticos y la performance profesional en el periodismo deportivo}

En la última década, los estudios han evolucionado, desde los clásicos estudios evaluativos sobre la importancia que los periodistas les daban a ciertas funciones, a un análisis sistemático de la forma en que los roles periodísticos se manifiestan en las noticias de todo el mundo (Hallin \& Mellado, 2018; Mellado \& Van Dalen, 2017; Mellado \& Vos, 2017; Stępińska, JurgaWosik, Adamczewska, Bartłomiej \& Dominika, 2016; Wang, Sparks, Lue $\&$ Huang, 2017). Dado que los estudios han demostrado una brecha inevitable entre los ideales profesionales y la práctica en el periodismo (Mellado, Hellmueller \& Donsbach, 2017), el estudio del desempeño de roles en el periodismo ha ganado relevancia en nuestro campo.

Los estudios sobre la puesta en práctica de los roles profesionales en la práctica periodística han analizado la performance del rol periodístico en tres dominios fundamentales: a) la presencia de la voz del periodista en las noticias, b) la relación que tiene el periodismo con los que están en el poder y c) la forma en que el periodismo se acerca a la audiencia (Mellado, 2015). Denominamos performance a la forma en que los roles periodísticos se materializan en el trabajo que realizan los profesionales de los medios de comunicación.

Específicamente, Mellado (2015) desarrollo un marco metodológico para identificar cómo los roles profesionales se plasman en las noticias, operacionalizando seis roles profesionales independientes, pero no mutuamente excluyentes, dentro de estos tres dominios. El primero aborda la postura pasivo-activa de los periodistas en sus informes, midiendo la presencia de sus voces en las narrativas que crean. El segundo dominio aborda la posición de los periodistas en relación con los que están en el poder, lo 
que les otorga dos roles profesionales independientes: el leal y el vigilante. Mientras que el rol vigilante o watchdog tiene como objetivo fiscalizar las acciones de los que están en el poder, el leal retrata a las élites bajo una luz positiva, o busca el fortalecimiento de la unidad nacional. El tercer dominio y final, se refiere a cómo el periodismo se dirige a la audiencia a través de tres roles profesionales independientes. El rol cívico se centra en la conexión entre el periodismo, la ciudadanía y la vida pública, acercándose a la audiencia como ciudadanos. El rol de servicio combina los derechos y los intereses propios de la audiencia, proporcionando información, conocimiento y asesoramiento sobre bienes y servicios que las audiencias pueden aplicar en su vida cotidiana. Finalmente, el rol de infotainment proporciona entretenimiento y relajo, y ve al público como espectador, a medida que sus experiencias emocionales se convierten en el centro de atención.

Hasta ahora, la presencia de estos roles profesionales no ha sido analizada en detalle con el objetivo de establecer si existen diferencias relevantes con otras secciones de noticias de los medios de comunicación. Además, los trabajos sobre roles profesionales se han concentrado en el estudio de una sola plataforma, en vez de comparar múltiples plataformas de medios (Mellado, Hellmueller \& Donsbach, 2017).

P3: ¿Existen diferencias significativas en la presencia de los roles profesionales (intervencionista, vigilante, leal, servicio, infoentretenimiento y cívico) en las noticias de la secciones de deportes y las generadas por otras secciones de los medios?

P4: ¿Existen diferencias significativas en la presencia de distintos roles profesionales en la cobertura deportiva entre la prensa escrita, los medios online, la televisión y la radio?

\section{El contexto chileno}

Chile se ha ganado la reputación de ser uno de los países más desarrollados y estables de América Latina. El país ha logrado importantes avances hacia la reducción de la pobreza desde el fin de la dictadura militar en 1990, y su democracia y su economía de mercado han sido elogiadas como una de las 
más fuertes de la región, hasta el punto de haber sido invitado a integrarse al grupo de los países de la Organización para la Cooperación y el Desarrollo Económicos (OCDE). Junto con su desarrollo económico, Chile ha logrado grandes avances en el acceso y uso de las tecnologías de la información y de la comunicación (TIC). Los usuarios de internet pasaron del 16,6 \% de la población en 2000 al $78 \%$ en 2016, alcanzando los niveles más altos de acceso a internet en América Latina (Pew Research Center: Journalism \& Media staff, 2005).

En lo que respecta a la prensa, los periódicos independientes y regionales casi han desaparecido, mientras que los dos principales conglomerados de periódicos (El Mercurio y Copesa) han crecido hasta formar un fuerte duopolio. Juntos, ambos grupos controlan los únicos cuatro periódicos distribuidos a nivel nacional, y casi el $90 \%$ de los periódicos regionales, todos los cuales están en el extremo derecho y conservador del espectro político.

En el paisaje digital, se ve una mayor variedad de puntos de vista, ya que periódicos digitales como El Mostrador (La Plaza S. A.), Terra (Terra Networks S. A.) o El Dínamo (Ediciones Giro País) han alcanzado altos niveles de tráfico. Sin embargo, en este ámbito, los dos principales conglomerados de prensa escrita, en especial El Mercurio, tienen una fuerte presencia.

En el caso de la televisión, el canal de televisión pública (TVN) ha vivido un fuerte declive en los últimos años y los demás canales de televisión abierta (Mega, Chilevisión y Canal 13) son propiedad de grandes grupos empresariales chilenos o extranjeros.

Finalmente, en la industria radial, se observa una presencia importante de actores extranjeros. Sin embargo, la producción de contenidos noticiosos está dominado por dos emisoras nacionales: Cooperativa y Bío Bío.

\section{Método}

\section{Muestra}

Se realizó un análisis de contenido de noticias de los medios nacionales más importantes de prensa impresa, online, canales de televisión y emisoras de 
radio de Chile. La muestra se recogió durante 2015 y en todos los medios se utilizaron los mismos días y unidades de análisis. De esta forma, dentro de cada medio, las unidades muestreadas fueron las ediciones seleccionadas, mientras que la unidad de análisis fue la noticia.

Los criterios para seleccionar los medios fueron el tamaño y el alcance de la audiencia, la orientación de la audiencia, la propiedad y el nivel de influencia en el establecimiento de la agenda. Los medios impresos elegidos fueron Las Últimas Noticias que representa a la prensa popular (N $=710)$ y $L a$ Tercera representa a la prensa elitista $(\mathrm{N}=1023)$, ambos entregando información de interés general con circulación nacional. Dentro de las noticias digitales, dos de los medios de comunicación en línea con mayor audiencia, El Mostrador $(\mathrm{N}=225)$ y El Dínamo $(\mathrm{N}=182)$, fueron analizados en su contenido. Para ser considerados, ambos cumplieron el criterio de ser puntos de venta digitales nativos en lugar de la versión digital de un medio tradicional impreso. En la televisión, se seleccionó el canal público TVN $(\mathrm{N}=362)$ y el canal privado Chilevisión $(\mathrm{N}=364)$. Por último, se incorporaron en la medición las dos principales radios informativas del país: Cooperativa $(\mathrm{N}=571)$ y Bío Bío $(\mathrm{N}=777)$.

El cronograma para el análisis de contenido fue el año completo de 2015. Al usar el método de semana construida, se seleccionó una muestra estratificada sistemática para cada medio seleccionado. En cada medio de comunicación, se seleccionaron al azar un lunes, martes, miércoles, jueves, viernes, sábado y un domingo de cada semestre del año. Dentro de cada una de las ediciones seleccionadas se analizaron todos los artículos de noticias publicados, los reportajes y las entrevistas.

\section{Medidas}

En la investigación, se utilizó la instrumentalización propuesta y validada por Mellado (2015), Mellado \& Van Dalen (2017) y Mellado et al. (2017), adaptando la operacionalización de los indicadores diseñados inicialmente para el formato de medios impresos a medios online, de radio y de televisión. 
Los seis roles periodísticos se examinaron a través de la presencia explícita de indicadores específicos en el contenido de las noticias. Cada uno de estos indicadores se codificó de acuerdo con la presencia (1) o ausencia (0) de la característica medida. Para cada rol profesional, se generaron escalas de elementos múltiples después de realizar un análisis factorial confirmatorio dentro de cada dominio. Del mismo modo, se midieron variables independientes asociadas a la calidad del reporteo de las noticias, como el número de fuentes, la diversidad de puntos de vista y la entrega de información verificable.

El proceso de búsqueda de las ediciones y de los artículos de la muestra, así como la codificación de cada noticia, fue realizado por seis codificadores, que fueron capacitados por los investigadores, de acuerdo con el libro de códigos y la ficha de análisis creada para este fin. Para reducir el sesgo, la muestra se dividió aleatoriamente entre codificadores.

La prueba global de confiabilidad intercoder final, basada en el alfa (Ka) de Krippendorff, fue de 0,75.

\section{Estrategia de análisis}

Para responder a las preguntas de investigación ya planteadas, se compararon los resultados de las noticias deportivas con las otras dos secciones, las de política y de entretención. Se escogió la sección de política porque es la que tradicionalmente se ha estudiado con mayor profundidad en las investigaciones sobre periodismo y roles profesionales. Es en las páginas de política en las que se podrían observar con mayor facilidad los elementos normativos tradicionalmente asociados al periodismo, principalmente la vigilancia de los actores públicos.

Por otra parte, la sección de entretención es una sección que, al igual de la de deportes, está más alejada de las exigencias tradicionales que se hacen al periodismo. El objetivo de incluir las noticias de esta sección es observar si existe similitud en el tratamiento que se da a la cobertura en comparación con las noticias de deportes, o en cambio, si ambas tienen características diferenciadas, a pesar de estar alejadas de la cobertura de noticias duras y del escrutinio permanente del poder. 
Las noticias de las secciones de política, deportes y entretención representan el 49,9\% del total de noticias recolectadas en el estudio (que se distribuyen en 14 secciones de noticias distintas). De hecho, se trata de las tres secciones más numerosas de la muestra: la sección de deportes llega al $19,8 \%$ del total, la de política sigue con el 17,3 \% y la de entretención ocupa el tercer lugar en el listado con el 13,1\%. Debido a la decisión de centrar el estudio en estas tres secciones noticiosas (de política, de deportes y de entretención), el tamaño de la muestra con que se realizaron los análisis estadísticos se redujo de 4.214 a 2.118 casos.

Las preguntas de investigación planteadas fueron respondidas utilizando pruebas de Chi-cuadrado y ANOVA.

\section{Resultados}

En sintonía con los estudios más críticos, los resultados de esta investigación muestran que las características de la cobertura noticiosa de la prensa deportiva es significativamente distinta de la de las secciones de Política, considerando aspectos como la cantidad de fuentes, la diversidad de puntos de vista y la entrega de información verificable al público. Sin embargo, sí se pueden observar similitudes importantes entre la cobertura de las noticias de deportes y las de entretención.

Del total de unidades analizadas, las noticias deportivas fueron las que presentaron el menor número de fuentes (personales o documentales), con solo un promedio de 1,57 por nota. La cifra es significativamente inferior a la alcanzada por la sección de política $(2,44)$, pero no presenta diferencias estadísticamente significativas con la sección de entretención $(1,84)$.

Este menor número de fuentes también tiene consecuencias en la diversidad de puntos de vista presentes en las notas de la sección de deportes (tabla 1). Solo en el 16,4 \% de las notas deportivas analizadas existe diversidad de puntos de vista de las fuentes. Es decir, que están presentes al menos dos fuentes con miradas distintas respecto de lo que se está informando. En la medición de esta característica, no se observan diferencias con la sección de entretención, pero sí existe una clara diferencia con 
la sección de política, ya que en esta última un 40,3 \% de las notas muestran distintos puntos de vista.

Por otra parte, en el $36 \%$ de las unidades analizadas en la sección de deportes, existe una ausencia de puntos de vista de las fuentes, frente a un $22,4 \%$ de la sección de entretención, y un 12,5 \% de la sección de política.

\section{Tabla 1. Diversidad de puntos de vista de las fuentes por sección $(\mathrm{N}=2,118)$}

\begin{tabular}{|c|c|c|c|}
\hline & $\begin{array}{c}\text { Ausencia de puntos de } \\
\text { vista de las fuentes (\%) }\end{array}$ & Cobertura unilateral (\%) & $\begin{array}{c}\text { Presencia de distintos } \\
\text { puntos de vista de las } \\
\text { fuentes (\%) }\end{array}$ \\
\hline Sección de deportes & 36,0 & 47,5 & 16,4 \\
\hline Sección de política & 12,5 & 47,3 & 40,3 \\
\hline Sección de entretención & 22,4 & 60,2 & 17,4 \\
\hline
\end{tabular}

$\chi^{2}$ de Pearson $=209,454 ;$ p-value $<0,001$.

Fuente: elaboración propia.

Respecto de la entrega de evidencia comprobable, esto es, que la información esté apoyada en hechos efectivamente ocurridos o en fuentes documentales consultadas, esta investigación mostró que en el 62,5 \% de las notas deportivas existe más información verificable que no verificable (tabla 2). Esta cifra supera lo que sucede en las unidades noticiosas de la sección de entretención analizadas $(52,8 \%)$, pero que está por debajo de lo que ocurre en la sección de política $(66,9 \%)$.

\section{Tabla 2. Presencia de evidencia comprobable/datos duros por sección $(\mathrm{N}=1,792)$}

\begin{tabular}{|c|c|c|}
\hline & $\begin{array}{c}\text { Hay menos información verificable que } \\
\text { información no verificable }(\%)\end{array}$ & $\begin{array}{c}\text { Hay más información verificable que } \\
\text { información no verificable }(\%)\end{array}$ \\
\hline Sección de deportes & 37,5 & 62,5 \\
\hline Sección de política & 33,1 & 66,9 \\
\hline Sección de entretención & 47,2 & 52,8 \\
\hline
\end{tabular}

$\chi^{2}$ de Pearson = 24,174; p-value $<0,001$.

Fuente: elaboración propia. 
Nuestra segunda pregunta de investigación apuntaba a conocer si las diferencias en la cobertura que realizan las secciones de deportes, política y entretención se reproducen de igual forma en las distintas plataformas tecnológicas. Como se observa en la tabla 3 , desde el punto de vista del número de fuentes consultadas, la mayor parte de las plataformas repiten el resultado encontrado para el conjunto de noticias analizadas: un promedio más alto de fuentes en la sección de política que en las secciones de deportes y entretención ( $\sin$ que estas dos últimas presenten diferencias significativas entre ellas). La única plataforma que escapa a este patrón es la televisión, en que la sección que tiene un promedio mayor de fuentes por nota es la de entretención con 5,06.

\section{Tabla 3. Número promedio de fuentes por nota en cada sección}

\begin{tabular}{|l|c|c|c|c|}
\hline & Medios impresos & Medios online & TV & Radio \\
\hline Sección de deportes & $1,48^{\mathrm{a}}$ & $1,32^{\mathrm{a}}$ & $2,55^{\mathrm{a}}$ & $0,92^{\mathrm{a}}$ \\
\hline Sección de política & $2,47^{\mathrm{ab}}$ & $2,39^{\mathrm{ab}}$ & $3,07^{\mathrm{b}}$ & $2,31^{\mathrm{ab}}$ \\
\hline Sección de entretención & $1,66^{\mathrm{b}}$ & $1,32^{\mathrm{b}}$ & $5,06^{\mathrm{b}}$ & $1,04^{\mathrm{b}}$ \\
\hline F & 23,859 & 9,038 & 13,765 & 74,909 \\
\hline p-value $<0,05$ & Sí & Sí & Sí & Sí \\
\hline N & 970 & 234 & 313 & 601 \\
\hline
\end{tabular}

Nota: el uso de superíndices implica diferencias estadísticamente significativas en materia de diversidad de puntos de vista (tabla 4). También en las distintas plataformas se observa un resultado similar al del conjunto de la muestra. En todas las plataformas, son las noticias políticas las que muestran una mayor variedad de puntos de vista ( $34,8 \%$ en medios impresos, 43,2 \% en medios online, 44,1 \% en televisión y 43,5 \% en radio) superando ampliamente a las secciones de deportes y entretención. De hecho, la sección de deportes solo supera el $20 \%$ de notas con diversidad de puntos de vista en televisión. 
Tabla 4. Diversidad de puntos de vista de las fuentes por tipo de plataforma (porcentaje de notas con diversos puntos de vista)

\begin{tabular}{|c|c|c|c|c|}
\hline & Medios impresos & Medios online & TV & Radio \\
\hline Deportes & $17,7 \%$ & $14,6 \%$ & $21,8 \%$ & $10,4 \%$ \\
\hline Política & $34,8 \%$ & $43,2 \%$ & $44,1 \%$ & $43,5 \%$ \\
\hline Entretención & $20,2 \%$ & $6,7 \%$ & $20,8 \%$ & $11,6 \%$ \\
\hline$\chi^{2}$ de Pearson de Pearson & 51,698 & 46,696 & 21,209 & 101,612 \\
\hline p-value $<0,05$ & Sí & Sí & Sí & Sí \\
\hline $\mathrm{N}$ & 970 & 234 & 313 & 601 \\
\hline
\end{tabular}

Fuente: elaboración propia.

Respecto de la presencia de datos duros en cada una de las plataformas, es posible observar similitudes y diferencias con el comportamiento general de la muestra. En los medios impresos y la televisión, las noticias políticas lideran esta categoría con el mayor porcentaje de datos duros en sus notas. Sin embargo, esta tendencia general se revierte en el caso de la radio, en el que la sección con más notas con información verificable es la de entretención. Por último, en el caso de los medios online, no se aprecian diferencias significativas (tabla 5).

\section{Tabla 5. Presencia de evidencia comprobable/datos duros por tipo de plataforma (porcentaje de notas con más información verificable que no verificable)}

\begin{tabular}{|l|c|c|c|c|}
\hline & Medios impresos & Medios Online & TV & Radio \\
\hline Sección de deportes & $61,7 \%$ & $51,2 \%$ & $69,3 \%$ & $61,0 \%$ \\
\hline Sección de política & $64,1 \%$ & $63,6 \%$ & $83,3 \%$ & $70,0 \%$ \\
\hline Sección de entretención & $48,8 \%$ & $52,0 \%$ & $56,5 \%$ & $82,6 \%$ \\
\hline$\chi^{2}$ de Pearson & 18,470 & 3,364 & 7,418 & 8,197 \\
\hline p-value $<0,05$ & Sí & No & Sí & Sí \\
\hline N & 970 & 234 & 228 & 360 \\
\hline
\end{tabular}

Fuente: elaboración propia. 
La tercera y cuarta preguntas de investigación están relacionadas con la presencia de los roles profesionales en las distintas secciones y plataformas en que se difunde la información.

Considerando el total de la muestra, destaca una mayor presencia del rol intervencionista en la sección de deportes (en comparación con las otras secciones analizadas); una mayor presencia de los roles leal, de servicio e infoentretenimiento en las noticias de entretención; y, por último, una mayor preponderancia de los roles watchdog y cívico en la sección de política (tabla 6). En todos los casos analizados, las diferencias de la presencia de los roles entre las notas de las tres secciones son significativas.

$\mathrm{Al}$ revisar la presencia de los roles en las distintas plataformas, no se observan grandes cambios respecto de cómo se distribuyen en el total de la muestra. Lo más llamativo es cómo aumenta la presencia del rol intervencionista en las noticias de entretención que se difunden en televisión y radio. En todas las plataformas analizadas, las diferencias de la presencia de los roles entre las notas de las tres secciones son significativas.

\section{Tabla 6. Presencia de roles profesionales por sección en todo la muestra y por tipo de plataforma}

\begin{tabular}{|c|c|c|c|c|c|c|}
\hline & Intervencionista & Watchdog & Leal & Servicio & $\begin{array}{c}\text { Info } \\
\text { entretenimiento }\end{array}$ & Cívico \\
\hline \multicolumn{7}{|c|}{ Total } \\
\hline Sección de deportes & $0,33^{\mathrm{a}}$ & $0,03^{\mathrm{a}}$ & $0,03^{\mathrm{a}}$ & $0,01^{\mathrm{a}}$ & $0,16^{\mathrm{a}}$ & $0,02^{\mathrm{a}}$ \\
\hline Sección de política & $0,21^{\mathrm{a}}$ & $0,10^{\mathrm{ab}}$ & $0,01^{\mathrm{a}}$ & $0,01^{b}$ & $0,08^{\mathrm{a}}$ & $0,05^{\mathrm{ab}}$ \\
\hline Sección de entretención & $0,29^{a}$ & $0,03^{\mathrm{b}}$ & $0,04^{\mathrm{a}}$ & $0,10^{\mathrm{ab}}$ & $0,22^{\mathrm{a}}$ & $0,02^{\mathrm{b}}$ \\
\hline $\mathrm{F}$ & 45,671 & 128,053 & 39,429 & 136,937 & 112,064 & 33,938 \\
\hline $\mathrm{p}$-value $<0,05$ & Sí & Sí & Sí & Sí & Sí & Sí \\
\hline $\mathrm{N}$ & 2.118 & & & & & \\
\hline \multicolumn{7}{|c|}{ Medios impresos } \\
\hline Sección de deportes & $0,29^{a}$ & $0,03^{\mathrm{a}}$ & $0,03^{\mathrm{a}}$ & $0,01^{\mathrm{a}}$ & $0,18^{\mathrm{a}}$ & $0,02^{\mathrm{a}}$ \\
\hline Sección de política & $0,16^{\mathrm{ab}}$ & $0,09^{\mathrm{ab}}$ & $0,01^{\mathrm{ab}}$ & $0,02^{\mathrm{b}}$ & $0,09^{\mathrm{a}}$ & $0,06^{\mathrm{ab}}$ \\
\hline Sección de entretención & $0,26^{\mathrm{b}}$ & $0,03^{\mathrm{b}}$ & $0,03^{b}$ & $0,10^{\mathrm{ab}}$ & $0,23^{\mathrm{a}}$ & $0,01^{\mathrm{b}}$ \\
\hline $\mathrm{F}$ & 31,478 & 55,739 & 6,414 & 57,713 & 51,563 & 49,687 \\
\hline $\mathrm{p}$-value $<0,05$ & Sí & Sí & Sí & Sí & Sí & Sí \\
\hline $\mathrm{N}$ & 970 & & & & & \\
\hline
\end{tabular}




\begin{tabular}{|c|c|c|c|c|c|c|}
\hline & Intervencionista & Watchdog & Leal & Servicio & $\begin{array}{c}\text { Info } \\
\text { entretenimiento }\end{array}$ & Cívico \\
\hline \multicolumn{7}{|c|}{ Medios online } \\
\hline Sección de deportes & $0,30^{\mathrm{a}}$ & 0,06 & $0,02^{\mathrm{a}}$ & $0,05^{\mathrm{a}}$ & 0,14 & $0,02^{\mathrm{a}}$ \\
\hline Sección de política & $0,19^{\mathrm{a}}$ & $0,10^{\mathrm{a}}$ & $0,00^{\mathrm{b}}$ & $0,00^{\mathrm{b}}$ & $0,09^{\mathrm{a}}$ & $0,05^{\mathrm{ab}}$ \\
\hline Sección de entretención & 0,26 & $0,04^{\mathrm{a}}$ & $0,05^{\mathrm{ab}}$ & $0,11^{\mathrm{ab}}$ & 0,19 & $0,02^{\mathrm{b}}$ \\
\hline $\mathrm{F}$ & 5,975 & 8,591 & 19,197 & 21,704 & 11,906 & 5,333 \\
\hline $\mathrm{p}$-value $<0,05$ & Sí & Sí & Sí & Sí & Sí & Sí \\
\hline $\mathrm{N}$ & 234 & & & & & \\
\hline \multicolumn{7}{|c|}{$\mathrm{TV}$} \\
\hline Sección de deportes & $0,50^{\mathrm{a}}$ & $0,03^{\mathrm{a}}$ & $0,05^{\mathrm{a}}$ & $0,02^{\mathrm{a}}$ & $0,25^{\mathrm{a}}$ & $0,04^{\mathrm{a}}$ \\
\hline Sección de política & $0,36^{\mathrm{ab}}$ & $0,10^{\mathrm{ab}}$ & $0,03^{\mathrm{b}}$ & $0,03^{\mathrm{b}}$ & $0,15^{\mathrm{a}}$ & 0,09 \\
\hline Sección de entretención & $0,52^{\mathrm{b}}$ & $0,03^{\mathrm{b}}$ & $0,09^{\mathrm{ab}}$ & $0,12^{\mathrm{ab}}$ & $0,35^{\mathrm{a}}$ & $0,11^{\mathrm{a}}$ \\
\hline $\mathrm{F}$ & 9,052 & 16,686 & 6,333 & 15,480 & 13,290 & 6,977 \\
\hline p-value $<0,05$ & Sí & Sí & Sí & Sí & Sí & Sí \\
\hline $\mathrm{N}$ & 313 & & & & & \\
\hline \multicolumn{7}{|c|}{ Radio } \\
\hline Sección de deportes & $0,25^{\mathrm{a}}$ & $0,01^{\mathrm{a}}$ & $0,02^{\mathrm{a}}$ & $0,01^{\mathrm{a}}$ & $0,05^{\mathrm{a}}$ & $0,01^{\mathrm{a}}$ \\
\hline Sección de política & $0,25^{\mathrm{b}}$ & $0,09^{\mathrm{ab}}$ & $0,01^{\mathrm{a}}$ & $0,01^{\mathrm{b}}$ & $0,06^{\mathrm{b}}$ & $0,04^{\mathrm{a}}$ \\
\hline Sección de entretención & $0,34^{\mathrm{ab}}$ & $0,02^{\mathrm{b}}$ & $0,05^{\mathrm{a}}$ & $0,10^{\mathrm{ab}}$ & $0,13^{\mathrm{ab}}$ & 0,03 \\
\hline $\mathrm{F}$ & 3,712 & 47,079 & 21,821 & 42,703 & 13,315 & 15,975 \\
\hline p-value $<0,05$ & Sí & Sí & Sí & Sí & Sí & Sí \\
\hline $\mathrm{N}$ & 601 & & & & & \\
\hline
\end{tabular}

Nota: el uso de superíndices implica diferencias estadísticamente significativas.

Fuente: elaboración propia.

\section{Discusión}

Los resultados de este estudio permiten empezar a conocer con más detalles la forma en funciona el periodismo deportivo y los roles periodísticos con que pueden observar en su ejercicio (performance) un terreno que ha sido poco explorado por la investigación.

En primer término, el estudio permite examinar que hay marcadas diferencias entre las características de la información publicada en la secciones de deporte y de política. En la misma línea de las críticas que el periodismo deportivo ha recibido por su eventual falta de rigor y profundidad, los resultados muestran que los periodistas deportivos —en comparación 
con sus pares de Política - consultan un número menor de fuentes en cada una de sus notas, integran un número inferior de puntos de vista y presentan al público menos información verificable. Las diferencias recién descritas no se observan cuando comparamos las noticias deportivas con la sección de entretención, que también históricamente ha sido considerada como lejana a la función pública del periodismo y ha recibido críticas por su forma de trabajo.

En términos de roles periodísticos, también se observan diferencias relevantes entre las formas en que trabajan los profesionales de las secciones de política, deportes y entretención. Los roles profesionales vinculados a la conexión del periodismo con el ámbito público — como el control de autoridades o información para la participación en las decisiones comunes- son más altos en las noticias políticas, en las que encontramos la mayor presencia de los roles vigilante y cívico.

Por otra parte, la sección de deportes está lidera por la presencia del rol intervencionista y supera a la sección de política en el rol leal. La presencia de estos dos roles es consistente con algunas de las críticas recibidas por el periodismo deportivo en publicaciones anteriores. El alto nivel alcanzado por el rol intervencionista da cuenta de una elevada presencia de la voz de los periodistas, quienes en su trabajo informativo opinan, interpretan, hablan en primera persona, usan constantemente adjetivos y demandan soluciones. Se trata de un hallazgo en línea con la falta de un reporteo más riguroso y el uso de información comprobable (the toy department of news media). El nivel alcanzado por el rol leal también puede vincularse con otra de las críticas recibidas por el periodismo deportivo: su excesiva cercanía de las fuentes y el retrato positivo que tiende a hacer de ellas. Los resultados del estudio muestran que el periodismo deportivo trata a los actores más importantes de su área de forma más positiva que la prensa política lo hace con la suya. Además, es posible que en la prensa deportiva el rol leal se vea alimentado por elementos de patriotismo o comparación positiva de Chile con otros países, vinculados a eventos deportivos internacionales. De hecho, 2015 fue un año marcado por los buenos resultados obtenidos en eventos deportivos internacionales por la selección chilena de fútbol, como la obtención por primera vez de la Copa América. 
A diferencia del parecido que las secciones de deportes y entretención mostraron al medir las prácticas de reporteo (número de fuentes, diversidad de puntos de vista e información verificable), en roles su comportamiento es distinto. Las noticias de entretención se encuentran asociadas a una fuerte presencia del rol de servicio, lo que implica preocupación por la vida cotidiana de su público y una tendencia a mirar a los miembros de la audiencia como eventuales consumidores de distintos productos y servicios. La cobertura de la sección de entretención también lidera claramente la presencia del rol de infoentretenimiento, en el que se prioriza la información sobre escándalos, sensacionalismo y vida privada.

Finalmente, los análisis estadísticos realizados no muestran que la presencia de los roles observados en cada una de las tres secciones analizadas (deporte, política y entretención) tenga cambios relevantes al estudiar por separado las cuatro plataformas incorporadas en la investigación (prensa escrita, prensa online, televisión y radio).

Si bien este estudio es pionero en el análisis de los periodistas deportivos en Chile, no está exento de limitaciones. Por ejemplo, solo se consideró en el análisis medios de comunicación de alcance nacional (sin considerar medios orientados a la difusión local) y la evidencia analizada corresponde a la situación de un solo país (Chile). Las futuras investigaciones podrán hacerse cargo de estas limitaciones y, principalmente, indagar si los resultados observados en Chile se repiten en otros países, con el objetivo de poder comparar lo que sucede con las prácticas profesionales de los periodistas deportivos en distintos sistemas de medios.

\section{Referencias}

Atkinson, J. (2011). Performance journalism: A three-template model of television news. The International Journal of Press/Politics, 16(1), 102-129. DOI: https://doi.org/10.1177\%2F1940161210381646

Billings, A. (2010). La comunicación en el deporte. Barcelona, España: UOC. 
Boyle, R. (2017). Sports journalism: changing journalism practice and digital media. Digital Journalism, 5(5), 493-495. DOI: https://doi. org/10.1080/21670811.2017.1281603

Cassidy, W. P. (2017). Inching away from the toy department: Daily newspaper sports coverage of Jason Collins' and Michael Sam's coming out. Communication \& Sport, 5(5), 534-553. DOI: https://doi. org/10.1177\%2F2167479516642205

Cushion, S. (2012). The democratic value of news: Why public service media matter. Nueva York, EE. UU.: Palgrave Macmillan.

Díaz, M. y Mellado, C. (2017). Agenda y uso de fuentes en los titulares y noticias centrales de los medios informativos chilenos. Un estudio de la prensa impresa, online, radio y televisión. Cuadernos.info, 40, 107-121. DOI: https://doi.org/10.7764/cdi.40.1106

Donsbach, W. (2008). Journalists' role perception. En W. Donsbach (Ed.), The international encyclopedia of communication, Vol.6, 2605-2610. Malden, EE. UU.: Blackwell.

Eide, M. \& Knight, G. (1999). Public/private service: Service journalism and the problems of everyday life. European Journal of Communication, 14(4), 525-547. DOI: https://doi.org/10.1177\% 2F0267323199014004004

English, P. (2016). Mapping the sports journalism field: Bourdieu and broadsheet newsrooms. Journalism, 17(8), 1001-1017.DOI: https:// doi.org/10.1177\%2F1464884915576728

English, P. (2017). Cheerleaders or Critics? Australian and Indian sports journalists in the contemporary age. Digital Journalism, 5( 5), 532548. DOI: https://doi.org/10.1080/21670811.2016.1209082

Farrington, N., Kilvington, D., Saeed, A. \& Price, J. (2012). Race, racism and sports journalism. Londres, RU: Routledge. 
Franks, S. \& O'Neill, D. (2016). Women reporting sport: Still a man's game? Journalism, 17(4), 474-492. DOI: https://doi. org/10.1177/1464884914561573

Garrison, B. \& Salwen, M. (1989). Newspaper sports journalists: A profile of the "profession". Journal of Sport and Social Issues, 13(2), 5768. DOI: https://doi.org/10.1177\%2F019372358901300201

Gómez Bueno, J. (2013). Ética, responsabilidad y observación de los códigos deontológicos en el periodismo deportivo (Tesis de doctorado, Universidad de Murcia, Murcia, España).

Hallin, D. C. \& Mellado, C. (2018). Serving consumers, citizens, or elites: Democratic roles of journalism in chilean newspapers and television news. The International Journal of Press/Politics, 23(1), 24-43. DOI: https://doi.org/10.1177\%2F1940161217736888

Hanitzsch, T. (2007). Deconstructing journalism culture: Toward a universal theory. Communication Theory, 17(4), 367-385. DOI: https:// doi.org/10.1111/j.1468-2885.2007.00303.x

Hardin, M. \& Whiteside, E. (2009). Token responses to gendered newsrooms: Factors in the career-related decisions of female newspaper sports journalists. Journalism, 10(5), 627-646. DOI: https:// doi.org/10.1177\%2F14648849090100050501

Márquez-Ramírez, M. \& Rojas Torrijos, J. L. (2017). ¿Periodismo deportivo pasivo o proactivo? La cobertura del FIFAGate en la prensa deportiva de México y España. Cuadernos.info, 40, 173-188. DOI: https://doi.org/10.7764/cdi.40.1009

Mellado, C. (2015). Professional roles in news content: Six dimensions of journalistic role performance. Journalism Studies, 16(4), 596-614. DOI: https://doi.org/10.1080/1461670X.2014.922276 
Mellado, C. \& Van Dalen, A. (2017). Challenging the citizen-Consumer journalistic dichotomy: A news content analysis of audience approaches in Chile. Journalism \& Mass Communication Quarterly, 94(1), 213-237. DOI: https://doi.org/10.1177/1077699016629373

Mellado, C. \& Vos, T. (2017). Conceptualizing journalistic role performance across news platforms. En C. Mellado, L. Hellmueller y W. Donsbach (Eds.), Journalistic role performance: Concepts, contexts and methods (pp. 106-124). Nueva York, EE. UU.: Routledge.

Mellado, C., Hellmueller, L. \& Donsbach, W. (Eds.) (2017). Journalistic role performance: Eoncepts, contexts, and methods. Nueva York, EE. UU.: Routledge.

Mellado, C., Hellmueller, L., Márquez-Ramírez, M., Humanes, M. L., Sparks, C., Stepinska, A., ...Wang, H. (2017). The hybridization of journalistic cultures: A comparative study of journalistic role performance. Journal of Communication, 67(6), 944-967. DOI: https:// doi.org/10.1111/jcom.12339

Poushter, J. (2016) 1. internet access growing worldwide but remains higher in advanced economies. Pew Research Center. Recuperado de http://www.pewglobal.org/2016/02/22/internet-accessgrowing-worldwide-but-remains-higher-in-advanced-economies/

Pew Research Center: Journalism \& Media staff. (2005). Box scores and bylines: A snapshot of the newspaper sports page. Recuperado de http:// www.journalism.org/2005/08/22/box-scores-and-bylines/

Rowe, D. (2004). Sport, culture \& media: The unruly trinity. Londres, RU: McGraw-Hill.

Rowe, D. (2007). Sports journalism: Still the toy department' of the news media? Journalism, 8(4), 385-405. DOI: https://doi. org/10.1177\%2F1464884907078657 
Salwen, M. B. \& Garrison, B. (1987). Sports and Politics: Los Angeles Times' Coverage of the 1984 Summer Olympic Games. Newspaper Research Journal, 8(2), 43-51. DOI: https://doi.org/10.1177\% 2F073953298700800205

Schultz-Jørgensen, S. (2005). The World's Best Advertising Agency: The Sports Press. The House of Monday Morning, 37. Recuperado de http://www.playthegame.org/upload/sport_press_survey_ english.pdf

Solves, J., Sánchez, S. \& Ruiz, I. (2016). The prince and the pauper: Journalistic culture and Paralympic Games in the Spanish print press. Journalism. DOI: https://doi.org/10.1177/1464884916671894

Stępińska, A., Jurga-Wosik, E., Adamczewska, K., Bartłomiej, S. \& Dominika, N. (2016). Journalistic role performance in Poland. Środkowoeuropejskie Studia Polityczne, 2, 37-51. Retrieved from http://hdl.handle.net/10593/15465

Sugden, J. \& Tomlinson, A. (2007). Stories from planet football and sportsworld: Source relations and collusion in sport journalism. Journalism Practice, 1(1), 44-61. DOI: https://doi. org/10.1080/17512780601078860

Surface, B. (1972). The shame of the sports beat. Columbia Journalism Review, $10(5)$, 48-55.

Valenzuela, S. y Arriagada, A. (2009). Competencia por la uniformidad en noticieros y diarios chilenos, 2000-2005. Cuadernos de Información, (24), 41-52. DOI: https://doi.org/10.7764/cdi.24.33

Wang, H., Sparks, C., Lue, N. \& Huang, Y. (2017). Differences within the mainland Chinese press: A quantitative analysis. Asian Journal of Communication, 27(2), 154-171. DOI: https://doi.org/10.1080 /01292986.2016.1240818 
Weedon, G., Wilson, B., Yoon, L. \& Lawson, S. (2018). Where's all the 'good' sports journalism? Sports media research, the sociology of sport, and the question of quality sports reporting. International Review for the Sociology of Sport, 53(6), 639-667. DOI: https:// doi.org/10.1177\%2F1012690216679835

Zelizer. B. (2017). What journalism could be. Cambridge, RU: Polity Press. 